\title{
Tracking error analysis of primary mirror in Linear Fresnel heat collecting field
}

\author{
Zhang ZhiYong ${ }^{1,2}$, Kong LingGang ${ }^{1}$, Fan DuoJin ${ }^{1}$, Yao XiaoMing ${ }^{1}$ \\ ${ }^{1}$ Engineering Research Center for Photothermal energy storage integrated energy system, Lanzhou Jiaotong University, Lanzhou \\ 730070 ; \\ 2. Key Laboratory of Opto-Technology and Intelligent Control Ministry of Education, Lanzhou Jiaotong University, Lanzhou 730070;
}

\begin{abstract}
Aiming at the tracking error of the primary mirror in the Linear Fresnel concentrated light and heat field, according to the structural characteristics of the Linear Fresnel concentrating and heat collecting system, the analysis model of the system concentrating error is established. By analyzing the mirror shape error of the Linear Fresnel system, the installation error of the secondary mirror (CPC), the errors of the north-south layout of the mirror field, the deviation of the primary mirror rotation axis and the deviation of the temperature drift of the angle sensor, and the factors were calculated by MATLAB simulation. The concentrating error factors affecting the Linear Fresnel concentrating and heat collecting system are analyzed quantitatively. From the simulation results: during the whole day, the influence of the north-south layout deviation of the mirror field and the deviation of the primary mirror rotation axis on the tracking accuracy is time-varying, and the influence is greater in the midday period. During the whole year, the influence factors of the north-south deviation of the mirror field and the temperature drift of the angle sensor on the tracking accuracy are incident, and the influence is greater in winter and less in summer. The installation error of CPC has a constant influence on the tracking accuracy.
\end{abstract}

\section{Introduction}

With the excessive consumption of fossil energy and the worsening of global environmental problems, the development of renewable and clean energy has become a major trend ${ }^{[1-3]}$. Solar energy has the advantages of clean, pollution-free and renewable. It is an ideal renewable energy. The best way to use solar energy is to collect light and heat ${ }^{[4,5]}$.According to the concentrating form, the solar concentrating system mainly includes trough type, Linear Fresnel type, dish type and tower type ${ }^{[6,7]}$,in various forms of solar thermal utilization, Linear Fresnel concentrator is characterized by simple structure, low wind resistance, low cost, high land volume ratio, uniform concentration, modularization, and scale design ${ }^{[8-10]}$, and is gradually becoming the most competitive form of photo-thermal power generation.

The Linear Fresnel solar collector system consists of three parts: reflector, receiver and control system. Heat collecting island is the energy supply unit of solar photo-thermal power station, and its concentrating effect has a very important influence on the generating capacity of the power station. The concentrating effect of heat island is mainly reflected in the production and processing precision of reflector. The engineering installation precision and the tracking precision of sun tracking system. For commercial plants, manufacturing error, installation error of the key components engineering ultimately reflected in the control system of the tracking error. It is very significant for linear focused solar energy utilization to reduce the inherent error of the heat collection system body, reduce the overflow loss of light and control the tracking error within an acceptable range by means of the optimization design of key parts and the precise positioning layout of mirror field.

Fraunhofer $I_{S E}{ }^{[11]}$ adopts two different dynamic testing methods to improve the performance of the Linear Fresnel primary mirror, making the average absolute error of the surface profile of the primary mirror less than $0.0098 \mathrm{mrad}$; Annie Hofer $\mathrm{A}^{[12]}$ used fringe reflection method and temperature compensation strategy to complete the quality control of the Linear Fresnel primary mirror surface; Chenglong Wang ${ }^{[13,14]}$ designed a high convergence rate Linear Fresnel type CPC receiver suitable for high temperature media. By studying the influence of maximum receiving half angle and interception ratio on CPC performance, it was concluded that the $\mathrm{CPC}$ opening width with the maximum receiving half angle of $45^{\circ}$ and interception ratio of 0.75 was $475.49 \mathrm{~mm} ; \mathrm{Ma} \mathrm{Jun}^{[15]}$ discussed the effect of shadows and occlusion on the optical efficiency of the Linear Fresnel convergent lens field system; By comparing several commonly used solar position calculation methods, Chunxu $\mathrm{Du}^{[16,17]}$ summarized a high-precision solar position calculation method with the maximum error of height angle and azimuth angle of $0.05^{\circ}$ and $0.13^{\circ}$. Research teams at home and abroad have done

E-mail: zhangzy@mail.lzjtu.cn 
important research on the structure design, performance optimization and quality control of Linear Fresnel system, For Fresnel commercial power plant, the tracking accuracy of primary reflector is very important for the thermal performance of the system and the safety of collector. There is no operating experience of large salt Fresnel photo-thermal power station in the world, and the research of tracking error in actual operation is rarely involved by experts and scholars. On the tracking error of the Linear Fresnel type concentrated heating system problems, combining with the characteristics of the structure of the Linear Fresnel system, and the Linear Fresnel concentrated heating system error analysis model is established, through matlab simulation, the effect factors of Fresnel system tracking accuracy is analyzed, and in order to reduce the tracking error of the Linear Fresnel type solar collector system, improving the efficiency of the Linear Fresnel commercial plant.

\section{Structure of the Linear Fresnel concentrated light and heat syste}

\subsection{Linear Fresnel set heat field structure}

Linear Fresnel type set two arrangement field from east-west and south-north ${ }^{[18]}$, The primary mirror consists of a flat or micro - arc mirror, under the tracking control device driver, direct sunlight reflection to the secondary mirror (CPC) receiver, sunlight is collected by CPC and projected onto the collector tube, a schematic diagram of the Linear Fresnel type solar system with north-south layout ${ }^{[19]}$ is shown in figure 1 . The calculation formulas of

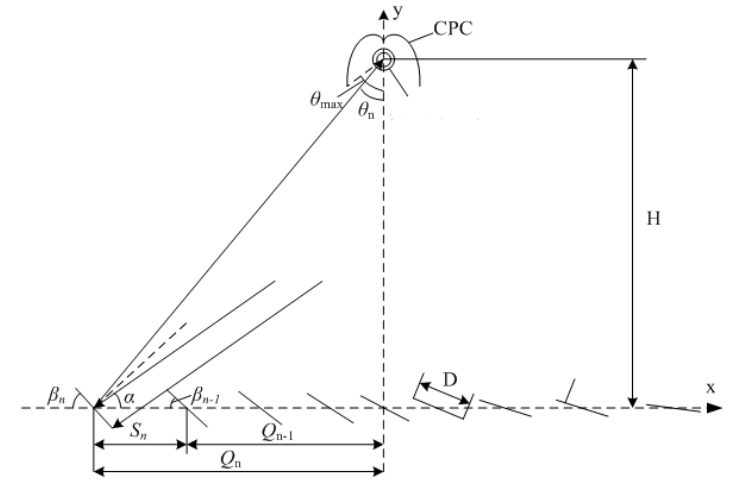

Fig.1 Linear Fresnel collector

\section{Analysis of tracking angle error of Linear Fresnel concentrator system}

\subsection{Primary mirror type error}

The primary mirror of the Linear Fresnel system usually has two structures: micro-arc and planar. The application of high temperature molten salt medium makes the heating system need to provide a higher multiples condenser, in order to obtain higher multiples condenser, slightly curved mirror to use more and more. Solar heating system can be thought of as large precision optical the tracking angles of the east and west mirrors of the heat collecting unit are shown in Formula 1 and 2.

$$
\begin{gathered}
\beta n_{-} e=\frac{(\alpha-a \tan (H / Q n))}{2}-\Pi / 2, \mathrm{n}=1,2, \cdots 10 \\
\beta n_{-} w=\frac{(\alpha+a \tan (H / Q n)-180)}{2}, n=1,2, \cdots 10
\end{gathered}
$$

In the equation, $\beta n_{-} e$ : the angle of the east columns, $\beta n \_w$ : the angle of the west columns, $H$ : the vertical distance from the CPC secondary reflector to the mirror placed horizontally, $Q n$ : the distance from the mirror array to the collector center, $\alpha$ : the angle of the sunlight.

\subsection{Structure of Linear Fresnel system driving device}

Considering the construction cost of the system, the complexity of the tracking control system and the cost of daily operation and maintenance, the multi-column Linear Fresnel primary reflector adopts the same set of driving device to realize the sun-chasing rotation of the mirror column. The multi-column Linear Fresnel mirror achieves positive and negative rotation by connecting rod mechanism according to the principle of parallelogram structure. The driving device, the tilt sensor and the controller together constitute a closed-loop control system, which completes the sun tracking control of the mirror column. Schematic diagram of driving and connecting rod mechanism is shown in Figure 2.

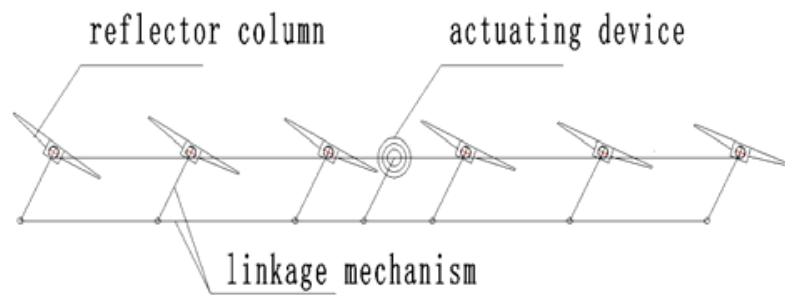

Fig.2 Linear Fresnel system drive and linkage mechanism

systems, Linear Fresnel primary reflector is a key component of optical system, a mirror surface error has a huge impact on system of concentrated effect. In order to achieve the ideal concentrating effect, the primary mirror type error must be controlled within a very small range, and the slope error detection of micro-arc mirror can be realized through fringe detection technology. In general, the primary mirror type slope error of the Linear Fresnel concentrating and heating system must be less than 10 $\operatorname{mrad}^{[20]}$. After the primary mirror is produced by the factory, it must be screened by the quality inspection system to ensure that the slope error of the surface of the primary mirror applied to engineering installation is less than $10 \mathrm{mrad}$. The detection result with slope error less 
than $3 \mathrm{mrad}$ and the focusing effect are shown in Fig. 3. In the error image of mirror detection results in Fig. 3, green indicates that the actual curvature of the mirror is

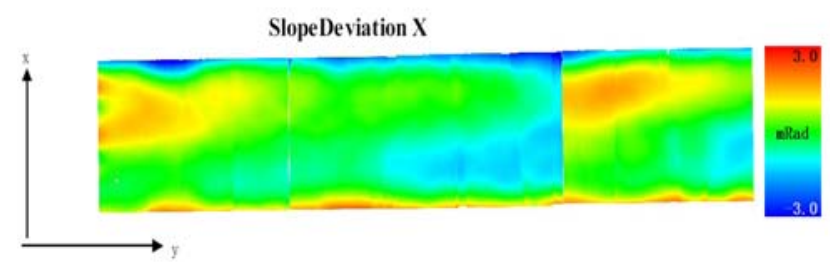

consistent with the designed curvature. The darker the color of red and blue parts, the greater the local curvature error of this part.

Fig.3 Mirror detection results and concentrating effect

\subsection{CPC installation error}

The Linear Fresnel system CPC secondary reflector is installed directly above the center of the collector mirror row column, The mounting height of CPC is determined according to the number of design columns of the primary mirror column, the spacing between mirrors, and the maximum accepted half angle of CPC. To comprehensively consider the construction cost of system, plant capacity and other factors, the installation of the CPC highly generally more than $10 \mathrm{~m}$, in the process of engineering construction, there are many structural parts and manufacturing processes, and the construction process is easily affected by many factors, such as environmental wind speed, foundation construction error, precision of measuring instrument and sense of responsibility of installation personnel. CPC installation errors are mainly divided into vertical installation errors and horizontal installation errors. The schematic diagram of installation error is shown in Fig. 4.

Simulation study on CPC installation error by MATLAB software found that the horizontal installation error of CPC had a significant influence on the tracking angle compared with the vertical installation error. When both horizontal and vertical installation errors were $50 \mathrm{~mm}$, the tracking angle error caused by CPC vertical installation error was about $0.025^{\circ}$, and the tracking angle error caused by CPC horizontal installation error was about $0.11^{\circ}$, the error is basically constant in the whole time period of tracking. Through matlab software simulation, the tracking angle deviation caused by horizontal and vertical installation errors is shown in Fig. 5 .

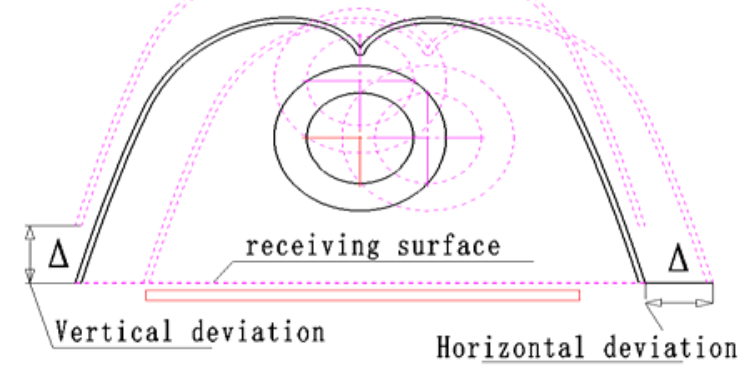

Fig.4 Schematic diagram of CPC horizontal and vertical installation errors
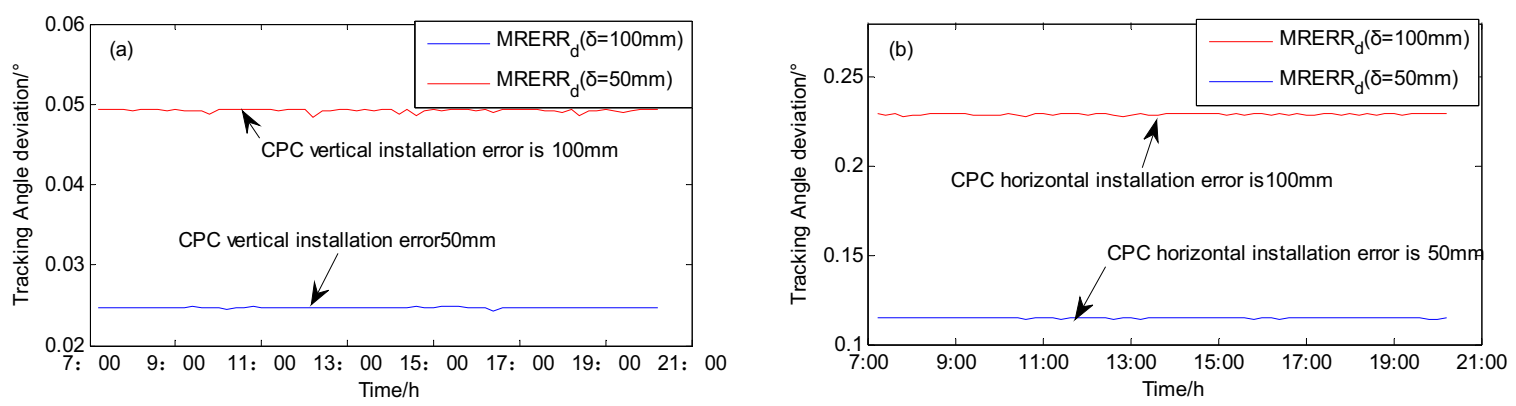

Fig.5 Tracking angle deviation due to $\mathrm{CPC}$ installation error.(a)Vertical installation error;

(b)Horizontal installation error

\subsection{South-north deviation of mirror field}

The calculation formula of the position of the sun is shown in Equation 3 and 4.

$$
\begin{gathered}
\hat{\alpha}=a \sin (\sin (\delta) * \sin (\text { latitude })+\cos (\delta) * \cos (\text { latitude }) * \cos (\omega)) \\
\gamma=a \cos (\sin (\hat{\alpha}) * \sin (\text { latitude })-\sin (\delta) / \cos (\hat{\alpha}) / \cos (\text { latitude }))
\end{gathered}
$$

In the equation, $\alpha$ : the solar altitude angle; $\delta$ : the declination of the sun; Latitude: Local solar dimension; 
$\omega$ : the solar hour angle; $\gamma$ : the azimuth of the sun; In reference 16 , by comparing several common solar position calculation methods, the author summarizes a high-precision solar position calculation method with the maximum error of height angle of $0.05^{\circ}$ and azimuth angle of $0.13^{\circ}$ respectively. After the height angle and azimuth angle of the sun are obtained, the angle between the incident ray of the sun in the east-west plane and the horizontal plane (that is, the solar incident angle often referred to in line-focused solar thermal applications) can be calculated by Formula 5 .

$$
\alpha=a \tan (\sin (\alpha) / \cos (\alpha) / \sin (\gamma))
$$

Where ${ }^{\alpha}$ : angle of the sunlight

Linear Fresnel concentrator with north-south layout, the tracking angle of the mirror column is symmetric with respect to noon in terms of time. The deviation between the north and the south of mirror field change tracking system "noon" time point. The actual layout of the mirror field is shown in Fig. 6.

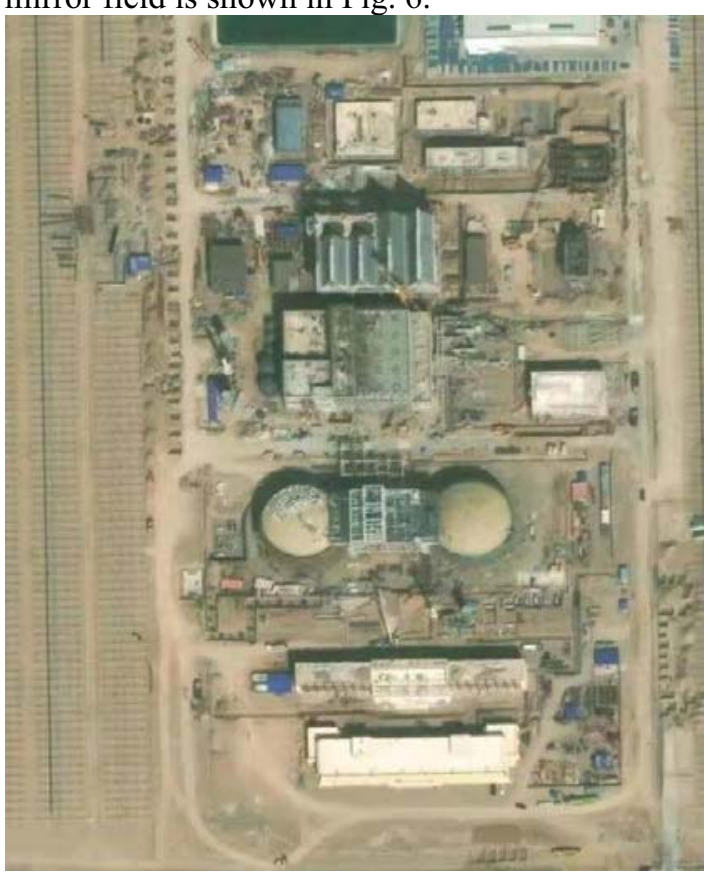

Fig.6 S atellite image of actual north-south deviation of heat collecting field

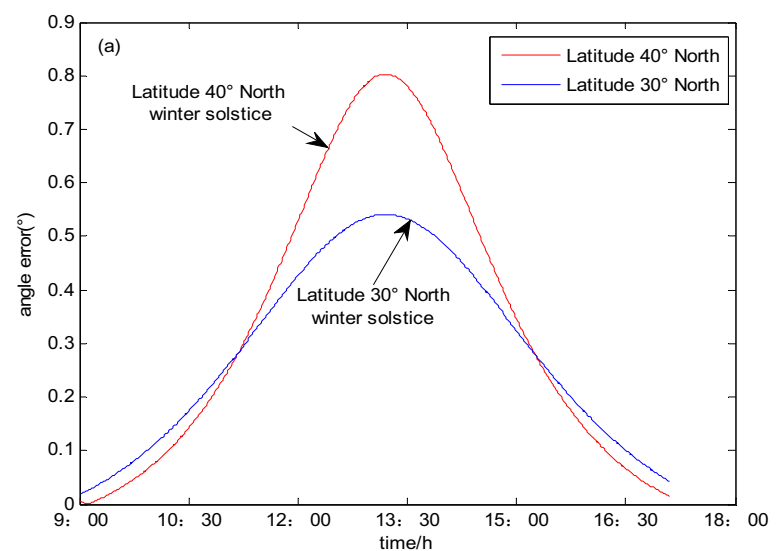

When the mirror field in the south by east errors, system operation can be thought of "noon" ahead of time arrival, mirror field south by west error, the system can be thought of running delay "noon" moment, so the sun incidence angle calculation formula of deformation for Formula 6 forms. Through MATLAB simulation, it is found that the tracking angle error caused by the north-south deviation of the Linear Fresnel mirror field is greatly different with the different latitude and seasons. According to the latitude analysis, the higher the latitude, the greater the deviation. According to the seasonal analysis, the winter solstice deviation is the largest, the summer solstice deviation is the smallest. According to the time analysis, the deviation is small in the morning and afternoon, and large at noon. The error curve of the whole day is roughly normally distributed at noon. When the mirror field has a north-south deviation, the tracking angle error with the latitude and seasonal variation curve is shown in Fig. 7.

$$
\begin{aligned}
& \alpha=180-a \tan \left(\sin (\alpha) / \cos (\alpha) / \sin \left(\mid \gamma+n_{-} s_{-} \text {off } \mid\right)\right), \omega<0 \& \gamma<0 \\
& \alpha=a \tan \left(\sin (\hat{\alpha}) / \cos (\hat{\alpha}) / \sin \left(\gamma+n_{-} s_{-} \text {off }\right)\right), \omega<0 \& \gamma>0 \\
& \alpha=180-a \tan \left(\sin (\hat{\alpha}) / \cos (\hat{\alpha}) / \sin \left(\gamma-n_{-} s_{-} \text {off }\right)\right), \omega>0
\end{aligned}
$$

$n_{-} s_{-}$off :South-north layout deviation of mirror field.

Fig.7 The tracking angle error caused by the south-north deviation of the mirror field. (a)Latitude $40^{\circ}$ north and $30^{\circ}$ north with the $\mathrm{N}$-S-offset is $0.8^{\circ}$; (b) Latitude $40^{\circ}$ north, N-S-offset is $0.8^{\circ}$. 


\subsection{Deviation of rotation axis of primary mirror}

Considering the cost and complexity of the Linear Fresnel tracking control system, Fresnel system multi-group mirrors track the sun through a set of driving device and connecting rod mechanism, a mirror micro-arc structure characteristics and the Linear Fresnel system running and maintenance requirements, make Fresnel system a reflection mirror and mirror has certain spacing between column shaft, when the mirror through the linkage to track the sun, the center of a mirror reflection time changing, cause specular reflection center and CPC changing the relative position of the center. For the long focal length optical system, the slight change in the position of the specular reflection center and the $\mathrm{CPC}$ receiving center will be amplified, causing the reflected spot of the primary mirror column to move laterally on the $\mathrm{CPC}$ receiving surface. The change of the reflection center trajectory when the primary mirror column rotates is shown in Figure 8(lest). Time-varying height and width distance are shown in Equation 7.

$$
\begin{array}{ll}
H_{d}=H+h-h^{*} \cos \left(\beta_{d}\right), & W_{d}=W-h^{*} \sin \left(\beta_{d}\right) \\
H_{x}=H+h-h^{*} \cos \left(\beta_{x}\right), & W_{x}=W+h^{*} \sin \left(\beta_{x}\right)
\end{array}
$$

where, $H_{d}, W_{d}$ :dynamic height and width of the eastern mirror column; $H_{x} W_{x}$ :dynamic height and width of the western mirror column; ${ }^{H},{ }^{W}$ : height

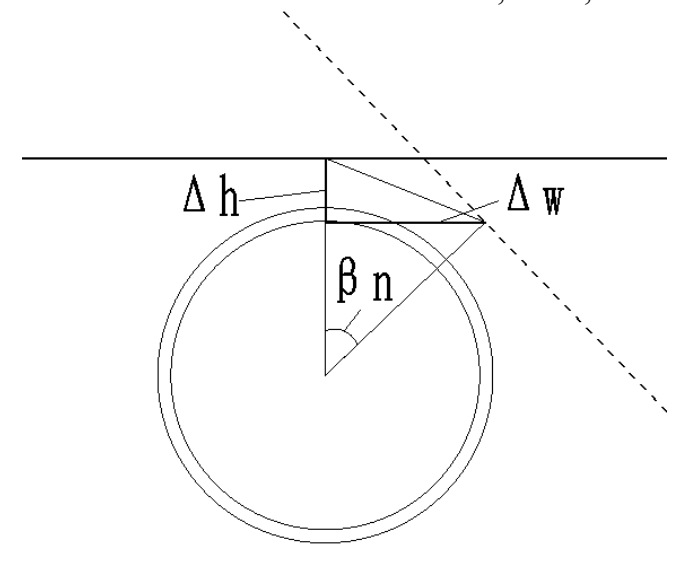

and width of mirror column when horizontal; $h$ : the distance between the rotation center of the mirror column and the mirror reflection center $\beta_{n}$ :dynamic dip angle during rotation of mirror column.

The curve of tracking angle error caused by the dynamic change of reflection center during rotation of each set of mirrors is shown in Figure 8(right). It can be seen from the figure that, in the tracking time period of the whole day, the tracking angle error introduced by the dynamic changes of height and width in the rotation process of the mirror column is relatively large in the morning and evening, and relatively small at noon. At the same time point, the tracking angle deviation introduced by different mirror elements is different due to different tilt angles lead to different column lens flare translational distance difference, the greater the rotation angle of mirror column, the greater the flare translation, when the column lens angle rotation to 0 degrees, tracking angle deviation disappear, for north and south to the layout of the Linear Fresnel system, multiple sets of mirror symmetrical about CPC receiving center east-west, when in the morning, on the west side mirror column rotation angle is bigger, in the afternoon, on the east side mirror column rotation angle is bigger, therefore, present in the reflected light spot on the surface of the CPC receiving, in the morning, on the east side mirror the width narrow reflected light spot, in the afternoon, the overall reflection spot of the west mirror line is narrow.

Fig.8 Schematic diagram of the running track of a mirror. (left)Rotation diagram;

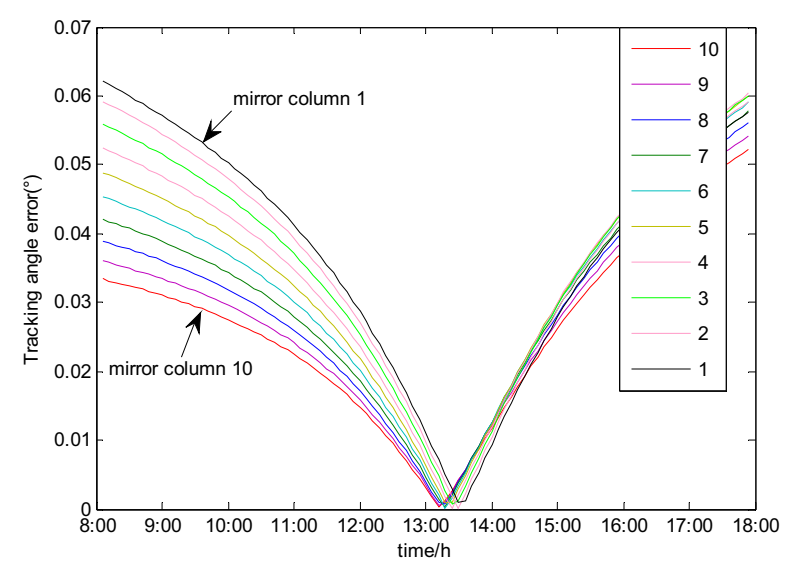

(right) Tracking errors at different mirror row

\subsection{Acuracy deviation of the angle sensor}

In Linear Fresnel tracking control system, the angle sensor is one of the key components to complete the drive motor closed-loop control. Manufacturing the angle sensor on the basis of the principle of micro-electro-mechanical inevitable environmental temperature change on the influence of the output angle, under normal circumstances, obliquity sensor output value for the corresponding relationship between temperature of $0.004^{\circ} /{ }^{\circ} \mathrm{C}$. Next to the abundant solar energy resources of the northwest region, the change of ambient temperature in winter and summer range up to $50{ }^{\circ} \mathrm{C}$, so the environment seasonal angle tracking error caused by the temperature change can be up to $0.2^{\circ}$. The corresponding error between the output value of the inclination sensor and the ambient temperature is shown in Fig. 9. 
0.5

0

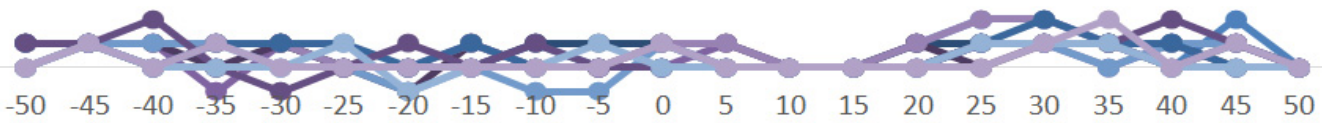

$-0.5$

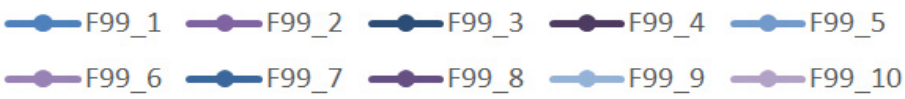

Fig.9 Influence of temperature on the output error of inclination sensor

Through the above analysis we can see that in the factors affecting the Fresnel system tracking error, the mirror surface error and the installation of the CPC to light gathering effect basic constant, when the reflection mirror and CPC receiving surface are installed, the tracking error caused by them will only affect the speckle by the slight deviation caused by the variation of the angle of the solar incident ray with seasonal changes. The influence of the south-north deviation of the field arrangement and the error introduced by the dynamic displacement of the rotating center of the mirror on the convergence effect is time-varying and nonlinear. The error caused by the north-south deviation is not only reflected in the seasonal variation, but also reflected in the hourly variation. The tracking deviation caused by the dynamic displacement of the rotating center of the mirror changes only with time and not with seasons.

\section{Conclusion}

Based on Linear Fresnel system tracking accuracy of the modeling analysis of influencing factors, it is concluded:

(1) The deviation of Linear Fresnel field layout system mirror effect on tracking accuracy in the year and throughout the day is the biggest, the greater the north deviation, the greater the error.

(2) The north-south deviation of the mirror field layout changes with the change of local latitude, the higher the latitude, the greater the error, the error presents a normal distribution trend on the time axis of the year and the time axis of the whole day.

(3) The tracking error caused by the distance between the mirror reflection center and the rotation axis is only reflected in the time axis of the whole day, and the greater the distance, the greater the error is.

(4) The installation errors of CPC in both vertical and horizontal directions will affect the tracking angle accuracy, and the installation errors in horizontal direction will have more obvious influence on the tracking accuracy. And the error is a constant value, does not change with time and season.

\section{Acknowledgments}

Fund project: National Energy Administration Project (China New Energy [2016]223);

Regional Science Foundation of China, No.51667013;
Major Science and Technology Special Project of Gansu Province, No.20ZD7GF001

\section{References}

1. Yuan Jiachun. Renewable Energy Development and Policy Analysis in China [J]. Chinese Market, 2018.

2. Wang Bo, LI Zhao-qian. Development Status and Trend of Global Renewable Energy [J]. China Prices, 2018, 000(005):44-47.

3. Li Qiming, ZHENG Jiantao, XU Haiwei, et al. Development of Linear Fresnel Solar Thermal Power Generation Technology [J].Solar Energy, 2012, 000(007):41-45.

4. Zhan Jing, WANG Zhifeng. Reflections on the Positioning of Solar Thermal Power Generation Technology in the New Generation of Energy System [J]. Progress in Electricity and Energy, 2018, 006(001): p.1-9.

5. Sun Yan, YU Hailong, ZHANG Changzheng. Analysis on the current situation and Development Trend of Solar power generation technology in China [J]. Smart Grid, 2014, 04(4):163-169.

6. Pan Kang, PANG Yuchang. Research review of Solar photovoltaic power generation technology [J]. China Solar Engineering, 2019, 000(003): P 35-36.

7. Liang Xinfeng. Research on the Development status of Solar Photovoltaic power Generation Technology [J]. China Strategic Emerging Industries, 2019, 000(038):27.

8. Huang F, Li L, Huang W. Optical performance of an azimuth tracking Linear Fresnel solar concentrator[J]. Solar Energy, 2014, 108(oct.):1-12.

9. Yu Qiu, Ya-Ling He, Ming Wu. A comprehensive model for optical and thermal characterization of a Linear Fresnel solar reflector with a trapezoidal cavity receiver[J]. Renewable Energy, 2016.

10. Bellos E, Tzivanidis C. Multi-criteria evaluation of a nanofluid-based Linear Fresnel solar collector[J]. Solar Energy, 2018, 163(MAR.):200-214.

11. Hofer A, Büchner, D, Kramer K, et al. Comparison of Two Different (Quasi-) Dynamic Testing Methods for the Performance Evaluation of a Linear Fresnel Process Heat Collector[J]. Energy Procedia, 2015, 69:84-95. 
12. Hofer A, Valenzuela L, Janotte N, et al. State of the art of performance evaluation methods for concentrating solar collectors[C]// American Institute of Physics Conference Series. AIP Publishing LLC, 2016:020010.

13. Wang Cheng-Cheng, MA Jun, Fan Du-wang, et al. CPC Simulation study for Linear Fresnel Concentrator $[\mathrm{J}]$.Infrared and Laser Engineering, 2015, 44(2):556-560.

14. Ma Jun, WANG Chenglong, Xia Yangjun. Composite Parabolic concentrator for Linear Fresnel Concentrator $[\mathrm{J}]$.Optical Precision Engineering, 2019.

15. Ma Jun, XIA Rongbin. Field shadow and Occlusion Analysis of Linear Fresnel Concentrator Based on Ray tracing [J].Journal of Lanzhou Jiaotong University, 2019, 38(4).

16. Du Chunxu, WANG Pu, Ma Zhongfang, et al.A high-precision Solar position algorithm [J]. Energy Engineering,2010,(2):41-44.

17. Du Chunxu, WANG Pu, MA Zhongfang, et al. A method for calculating the position of the Sun accurately with astrometric resume $[\mathrm{J}]$. Renewable Energy, 2010, 28(003):85-88.

18. Reda I, Andereas A. Solar position algorithm for solar radiation application[J]. Solar Energy, 2004, 76(5):577-589.

19. Wang Chenglong, Ma Jun, Fan Dowang. Mirror Field Arrangement and Optimization of Linear Fresnel Concentrator system [J].Optical Precision Engineering, 2015, 23(001):78-82.

20. Montecchi, Marco. Upgrading of ENEA Solar Mirror Qualification Set-up[J]. Energy Procedia, 2014, 49:2154-2161. 